\title{
Higher order oscillations of (3+1)D light bullets in linearly inhomogeneous cubic-quintic media
}

\author{
Jerzy Jasiński* \\ Faculty of Physics, Warsaw University of Technology, Koszykowa 75, 00-662 Warszawa
}

Received March 22, 2012; accepted March 28, 2012; published March 31, 2012

\begin{abstract}
In the paper the propagation is considered of $(3+1) \mathrm{D}$ axially-symmetric spatio-temporal pulses through cubic-quintic nonlinear Kerr-like with linear graded-index modulation. The analysis of propagation is performed by means of the variational method. The approximate solutions of Euler-Lagrange (EL) equations are obtained using multiple scale approximation up to second-order correction terms. The results are compared with a numeric solution of the nonlinear Schrödinger equation.
\end{abstract}

Let us consider an axially-symmetric (3+1)D light bullet propagating in a nonlinear cubic-quintic medium with axially-symmetric graded-index modulation of the linear index. Its envelope $U(x, y, z, t)=U(r, z, t)$ satisfies the nonlinear Schrödinger equation (NLSE):

$$
U_{z}-\frac{i}{2 k}\left(U_{r r}+\frac{U_{r}}{r}\right)+\frac{i k_{2} U_{t t}}{2}-i \varepsilon_{N L} U+\frac{i k \Omega^{2} r^{2} U}{2}=0
$$

(the index after the function denotes differentiation over the indicated coordinate and $\Omega$ modulation coefficient). The cubic-quintic permittivity equals:

$$
\varepsilon_{N L}=\varepsilon_{2}\left(|U|^{2}-|U|^{4} / I_{\text {sat }}\right) .
$$

Assume the field of the bullet as the product of the spatial $R(r, z)$ and the temporal $u(z, t)$ terms:

$$
U(r, z, t)=R(r, z) u(z, t)
$$

Because the nonlinearity $\varepsilon_{N L}$ couples $R$ and $u$, the field (3) does not satisfy NLSE (1). Nevertheless, we can treat it as an approximate solution in which $R$ satisfies the linear transverse part of Eq. (1) and describes Gaussian beam propagating in media with permittivity modulated by the last term of NLSE while $u$ satisfies the nonlinear part of Eq. (1) and describes a temporal soliton. Note that the parameters of this soliton are defined by the effective nonlinearity coefficient $\varepsilon_{2(e f f)}=\varepsilon_{2}|R|_{(\text {eff })}^{2}$ and the effective saturation $I_{\text {sat (eff) }}=I_{\text {sat }} /|R|^{2}($ eff $)$ depending on a constant value of $|R|^{2}=|R|_{(\text {eff })}^{2}$ enabling decomposition of NLSE [1].

Assume that the temporal and spatial width together with the bullet's peak the field change during propagation. To describe these changes we shall apply the variational method [2-7]. NLSE (1) corresponds to Lagrange density [2-4]:

$$
\text { *E-mail: jasinski@if.pw.edu.pl }
$$

$$
\begin{aligned}
L_{D}= & \frac{i\left(U U_{z}^{*}-U_{z} U^{*}\right)}{2}-\frac{k_{2} U_{t} U_{t}^{*}}{2}+\frac{U_{r} U_{r}^{*}}{2 k}+\frac{k \Omega^{2} r^{2}}{2} \\
& -\varepsilon_{2}\left(\frac{|U|^{4}}{2}-\frac{|U|^{6}}{3 I_{\text {sat }}}\right)
\end{aligned}
$$

As the trial function describing field $\mathrm{U}=U(r, z, t)$ we take:

$$
U=\frac{b}{\cosh (t / w)} \exp \left(-\frac{r^{2}}{2 a^{2}}+i\left(\beta+\alpha r^{2}+\theta t^{2}\right)\right)
$$

with the evolving peak field $b(z)$ temporal and spatial widths $w(z)$ and $a(z)$, propagating term $\beta(z)$, Gaussian curvature $\alpha(z)$ and chirp $\theta(z)$. The Lagrange function $L$ is obtained by the integration of $L_{D}$ with $U$ given by (5) over time. Doing so we arrive at [2-4]:

$$
\begin{aligned}
L= & 2 \pi b^{2} a^{2} w\left(\beta_{z}+\frac{\pi^{2} w^{2}\left(\theta_{z}-2 k_{2} \theta^{2}\right)}{12}-\frac{k_{2}}{6 w^{2}}+\frac{2}{2 k a^{2}}\right. \\
& \left.+a^{2}\left(\alpha_{z}+\frac{2 \alpha^{2}}{k}+\frac{k \Omega^{2}}{2}\right)-\frac{\varepsilon_{2} b^{2}}{6}+\frac{8 \varepsilon_{2} b^{4}}{135 I_{\text {sat }}}\right)
\end{aligned}
$$

Treating six evolving functions $\psi(z)$ in $U$ as generalized coordinates we can write six Euler-Lagrange equations: $d\left(\partial L / \partial \psi_{z}\right) / d z-\partial L / \partial \psi=0$. In order to simplify further calculations let us apply dimensionless quantities defined as follows:

$$
\begin{gathered}
Z=\Omega z, \quad \Delta=\frac{32 \Omega}{9 \varepsilon_{2} I_{\text {sat }}}, \quad \xi=-\frac{k_{2} \theta}{\Omega}, \quad \gamma=\frac{\alpha}{k \Omega}, \\
B=\sqrt{\frac{\left|\varepsilon_{2}\right|}{\Omega}} \frac{b}{2}, \quad A=\sqrt{k \Omega} a, \quad W=\sqrt{\frac{\Omega}{\left|k_{2}\right|}} w
\end{gathered}
$$

Moreover, to specify the type of the media let us consider positive nonlinearity $\varepsilon_{2}>0$ and negative group velocity dispersion $k_{2}<0$. One of the obtained EL equation can be integrating immediately giving:

$$
2 B^{2} A^{2} W=P
$$

The constant $\mathrm{P}$ that appears here we can identify as a quantity proportional to total power carried by the bullet:

$$
P=\frac{k\left|\varepsilon_{2}\right|}{4 \pi} \sqrt{\frac{\Omega}{\left|k_{2}\right|}} \cdot \int_{0}^{\infty} \int_{-\infty}^{\infty}|U|^{2} d t r d r
$$


Two another EL equations describe the evolution of the spatial and the temporal width. Although they contain the Gaussian curvature and the chirp, we can eliminate them using the remaining EL equations. Doing so we obtain:

$$
\begin{aligned}
& A_{\mathrm{ZZ}}=F(A, W) \\
& W_{\mathrm{ZZ}}=G(A, W)
\end{aligned}
$$

(the index containing a letter means differentiation over appropriate quantity) where functions appeared in the right-hand expressions of Eq. (8) are given by the expressions:

$$
\begin{gathered}
F=\frac{1}{A^{3}}-A-\frac{2 P}{3 W A^{3}}+\frac{\Delta P^{2}}{3 W^{2} A^{5}} \\
G=\frac{4}{\pi^{2}}\left(\frac{1}{W^{3}}-\frac{P}{W^{2} A^{2}}+\frac{\Delta P^{2}}{2 W^{3} A^{4}}\right)
\end{gathered}
$$

The equations (10) describe a more generalized case of the cubic-quintic medium than that discussed in [4].They have no general analytic solution, but we can report two special solutions: $(i)$ oscillating Gaussian beam of initial width $A(0)$ obtained for $W \rightarrow \infty$ :

$$
A(Z)=\sqrt{A(0)^{2} \cos ^{2} Z+\sin ^{2} Z / A(0)^{2}},
$$

and (ii) stationary solution $A=$ const $=A_{0}, W=$ const $=W_{0}$. Although the expressions for $A_{0}$ and $W_{0}$ given as functions of power $\mathrm{P}$ are very complicated, we can write them by explicit formulae as the functions of width $W_{0}$. Together with $B_{0}\left(W_{0}\right)$ following from Eq. (8) they give:

$$
\begin{gathered}
A_{0}=\sqrt{\frac{\sqrt{9 W_{0}^{4}+1}-1}{3 W_{0}^{2}}} \\
P=\frac{\left(\sqrt{9 W_{0}^{4}+1}-1\right)\left(W_{0}-\sqrt{W_{0}^{2}-2 \Delta}\right)}{3 \Delta W_{0}^{2}} \\
B_{0}=\sqrt{\frac{W_{0}-\sqrt{W_{0}^{2}-2 \Delta}}{2 \Delta W_{0}}}
\end{gathered}
$$

To describe a small deviation from stationary solutions we shall apply a multiple scale technique [8]. Let us write:

$$
\begin{gathered}
A(Z)=A_{0}+\delta A(Z)+\delta \delta A(Z) \\
W(Z)=W_{0}+\delta W(Z)+\delta \delta W(Z)
\end{gathered}
$$

where $|\delta \delta \psi| \ll|\delta \psi| \ll|\psi|$ for $\psi=A$ and $\psi=W$. Introducing (14) into (10)-(11) and expanding $F$ and $W$ into Taylor series we obtain two highest order equations $F\left(A_{0}, W_{0}\right)=0, G\left(A_{0}, W_{0}\right)=0$ determining a stationary solution (13). The first and the second order equations give:

$$
\begin{aligned}
& \delta A_{Z Z}=F_{A} \cdot \delta A+F_{W} \cdot \delta W \\
& \delta W_{Z Z}=G_{A} \cdot \delta A+G_{W} \cdot \delta W
\end{aligned}
$$

$$
\begin{gathered}
\delta \delta A_{Z Z}=F_{A} \delta \delta A+F_{W} \delta \delta W+\frac{F_{A A} \delta A^{2}}{2}+F_{A W} \delta A \delta W+\frac{F_{W W} \delta W^{2}}{2} \\
\delta \delta W_{Z Z}=G_{A} \delta \delta A+G_{W} \delta \delta W+\frac{G_{A A} \delta A^{2}}{2}+G_{A W} \delta A \delta W+\frac{G_{W W} \delta W^{2}}{2}
\end{gathered}
$$

The equations (15) describe small oscillations around the stationary solutions. Looking for a solution of the form $\delta \psi(Z)=\delta \psi(0) \exp (i \omega Z)$, we obtain:

$$
\begin{aligned}
-\omega^{2} \delta A & =F_{A} \delta A+F_{W} \delta W \\
-\omega^{2} \delta W & =G_{A} \delta A+G_{W} \delta W
\end{aligned}
$$

The condition for vanishing of the determinant of the system (17) gives to possible values of $\omega$ - two normal frequencies:

$$
\begin{aligned}
& \omega_{1}^{2}=-\frac{F_{A}+G_{W}}{2}-\frac{1}{2} \sqrt{\left(F_{A}-G_{W}\right)^{2}+4 F_{W} G_{A}} \\
& \omega_{2}^{2}=-\frac{F_{A}+G_{W}}{2}+\frac{1}{2} \sqrt{\left(F_{A}-G_{W}\right)^{2}+4 F_{W} G_{A}}
\end{aligned}
$$

Therefore the first-order corrections in the expansion (14) describe superposition of oscillations with frequency $\omega_{1}$ and $\omega_{2}$. Fig. (1) illustrate such oscillations - it contains the numeric solution of Eq. (1) obtained by BPM method.

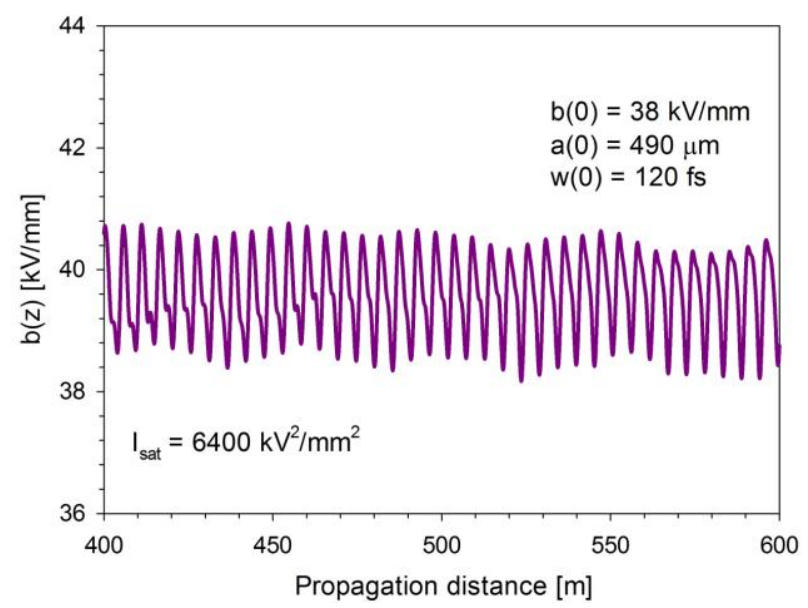

Fig. 1. Evolution of peak field in CQ medium - complex superposition of oscillations.

In Fig. 1 we can distinguish oscillations with a certain fundamental frequency modulated by a much smaller one. For initial values reported in the picture we have $P=$ $254.3 \mathrm{kV}^{2} \mathrm{ps}(=0.5533$ in dimensionless units $)$, which gives $\omega_{1}=0.0797 / \mathrm{m}$ and $\omega_{1}=1.1590 / \mathrm{m}$ calculated by means of Eq. (18) - and these values perfectly coincide with those observed in Fig. 1. But the picture of the bullet's evolution is much more complex - to analyse it let us perform the Fourier transform.

In Fig. 2 we see the Fourier transform of the bullet's peak field $b(z)$ together with the Fourier transform of 
spatial width $a(z)$ - at any distance it can be determined during calculations.
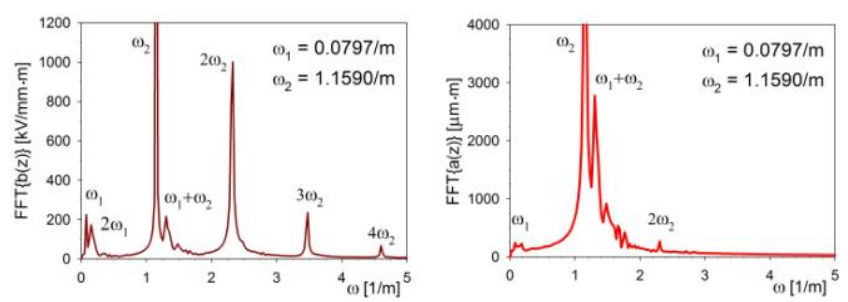

Fig. 2. Frequency domain of peak field and spatial width. Propagation distance between 400 and $600 \mathrm{~m}$. Parameters of medium and bullet the same as in Fig. 1.

We observe oscillations with more than two frequencies these additional frequencies are simple multiples and the sum of $\omega_{1}$ and $\omega_{2}$. A similar picture can be seen at the frequency domain of temporal width $w(z)-$ but its determination during calculation is much less accurate, so instead we shall analyse the Fourier transform of $b(z)-$ as in Fig. 2.

The quotients $\delta W(Z) / \delta A(Z)$ and $\delta B(Z) / \delta A(Z)$ are fixed during oscillations with normal frequency [3]:

$$
\frac{\delta W(Z)}{\delta A(Z)}=-\frac{F_{A}+\omega^{2}}{F_{B}}, \quad \frac{\delta B(Z)}{\delta A(Z)}=-\frac{B_{0}\left(F_{A}+\omega^{2}\right)}{2 W_{0} F_{B}}-\frac{B_{0}}{A_{0}}
$$

so for $\omega_{1}$ and $\omega_{2}$ they are different. Calculating them by means of Eq. (17) we obtain for the considered example $\delta B_{1} / \delta A_{1}=-6.70$ and $\delta B_{2} / \delta A_{2}=-0.396$ in dimensionless units. On the other hand, the height of the Fourier peak is proportional to the amplitude of oscillation at a given frequency, so we can find these quotients numerically. In this way $\left|\delta B_{1} / \delta A_{1}\right|_{\text {num }}=6.57$ and $\left|\delta B_{2} / \delta A_{2}\right|_{\text {num }}=0.410$. Therefore, the description of the bullet's evolution given in Fig. 1 as a superposition of two normal oscillations is quite accurate in the first order approximation.

The second order terms $\delta \delta \psi(Z)$ satisfy Eqs. (16). In the right hand expressions we distinguish the terms describing free oscillations with frequency $\omega_{1}$ and $\omega_{2}$. The remaining terms represent harmonic forces inducing stimulated oscillations with frequency $\omega_{1}, \omega_{1}+\omega_{2}$ and $2 \omega_{2}$. The amplitude of any component $\delta \delta \psi(Z)$ oscillating with a given frequency is proportional to appropriate force. In this way we can obtain the relation describing proportions between amplitudes of different components:

$$
\begin{gathered}
\frac{\delta \delta A_{1+1}}{\delta A_{1}^{2}}=\frac{1}{4 \omega_{1}^{2}}\left(\frac{G_{A A}}{2} q_{1}^{2}+G_{A W} q_{1}+\frac{G_{W W}}{2}\right) \\
\frac{\delta \delta A_{1+2}}{\delta A_{1} \delta A_{2}}=\frac{1}{\left(\omega_{1}+\omega_{2}\right)^{2}}\left(G_{A A} q_{1} q_{2}+G_{A W}\left(q_{1}+q_{2}\right)+\frac{G_{W W}}{2}\right) \\
\frac{\delta \delta A_{2+2}}{\delta A_{2}^{2}}=\frac{1}{4 \omega_{2}^{2}}\left(\frac{G_{A A}}{2} q_{2}^{2}+G_{A W} q_{2}+\frac{G_{W W}}{2}\right)
\end{gathered}
$$

where $q_{m}=\delta W_{m} / \delta A_{m}$.

The numeric values calculated from Eq. (20) give the height of the peaks $2 \omega_{1}$ and $2 \omega_{2}$ about 3 times smaller while the height of the peak $\omega_{1}+\omega_{2}$ is about 10 times smaller than that observed in Fig. 2 for oscillations of $a(z)$ (for oscillations of $b(z)$ the peak $2 \omega_{2}$ is also 10 times greater). The reason is simple - we are far beyond the approximation $|\delta \delta \psi| \ll|\delta \psi|$. For less power we obtain a much better situation, but most of higher order peaks will not be visible at the Fourier transform of numerically obtained functions $a(z)$ and $b(z)$. Moreover, the bullets carrying less energy propagate less stable - saturation (more important for greater power) stabilizes propagation. In fact, during propagation we observe a few additional elements not included in the description given in this paper:

(i) The deviation of the bullet's shape from that given by Eq. (5). We could assume a bit better trial function taking into account the temporal profile suitable for a cubicquintic medium [9], but such a shape contains more oscillating parameters

(ii) Third and even higher order harmonics

(iii) Coupling end energy transfer between oscillating components of close frequencies $\left(\omega_{1}, 2 \omega_{1}\right)$ and $\left(\omega_{2}, \omega_{1}+\omega_{2}\right)$

In spite of the above inaccuracies, the first order corrections agree with numerics very well - for instance the value of $\omega_{2}$ up to 3 significant digits $\left(\omega_{1}\right.$ one order worse). Therefore, the obtained relations (17)-(18) describe quite exactly the propagation up to a first order correction with respect to a stationary solution. Second order corrections give proper values of second order harmonics, but the amplitudes of these harmonics are evaluated rather qualitatively.

\section{References}

[1] G.P. Agrawal, Nonlinear Fiber Optics (Academic Press, San Diego 2001).

[2] J. Jasinski, Opto-Electron. Rev. 13, 129 (2005).

[3] J. Jasiński, M. Kościesza, Phot. Lett. Poland 2, 131 (2010).

[4] S. Raghavan, G.P. Agraval, Opt. Commun. 180, 377 (2000).

[5] V. Skarka, V.I. Berezhiani, R. Miklaszewski, Phys. Rev. E 56, 1080, (1997).

[6] D.E. Edmundson, R.H. Enns, Phys. Rev. A 51, 2491 (1995).

[7] D. Mihalache, D. Mazilu, L.-C. Crasovan, B.A. Malomed, F. Lederer, Phys. Rev. E 61, 7142 (2000).

[8] R.F. Abdullatif, R. Dewar, Int. J. Basic Appl. Sci. 11, 120 (2011).

[9] A. Biswas, S. Konar, Introduction to non-Kerr Law Optical Solitons (Chapman \& Hall, Boca Raton 2007). 